\title{
Development of nanoparticles for clinical use
}

\author{
"...it is necessary to establish more crossfunctional collaboration between experts in \\ the different relevant scientific areas..."
}

Keywords: drug approval • excretion $\bullet$ metabolism $\bullet$ nanoparticles $\bullet$ risk/benefit $\bullet$ toxicity

During recent years, there has been much interest in using nanoparticles (NPs) for delivery of therapeutic drugs or contrast agents. Many interesting in vitro and animal studies have been reported [1-3], and some NP-based products are already in clinical use. Iron-oxide containing NPs have been used as iron supplement or contrast agents in MRI for more than 20 years [4,5]. Albumin and liposomal-based products containing anticancer drugs have also been available for several years, and new NP-based products are in clinical trials $[2,6]$. The discrepancy between the large number of papers published about NPs and other drug candidates versus the few new drugs entering the market is heavily debated, and important discussions regarding what is needed to bring new products to the market have been published [6-9]. No doubt, there are many challenges to overcome before NPs may become common tools in clinical practice.

\section{Regulatory requirements \& costs for bringing new drugs to the market}

Guidelines for studies needed to obtain approval for marketing of new drugs or imaging agents are available from regulatory authorities and show that all new products for human use have to pass a long list of efficacy and safety studies [10]. Not only shortterm studies on the immediate impact of the injected NPs are needed, but also studies for longer periods of time are necessary to investigate whether there are any effects caused by the partly or fully degraded NPs. It is important to document that the NPs, unless they are small enough to be rapidly excreted in urine, are biodegradable so that nonendogenous constituents can be excreted. Clearly, this is not a problem for products such as liposomes or albumin-based NPs built up of endogenous substances, but it may be a challenge with many other NP-based products. The rates of biodegradation and excretion of nonendogenous substances will determine for how long the regulatory studies have to be performed [10]. If traces of NPs are left for a long time, the toxicity studies have to last longer and the cost of the development studies are thereby increased. Quite a number of studies are needed for obtaining approval of new drugs, and the following list should be regarded only as an indication of what is required for approval of intravenously injected NPs:

- Physiochemical characterization of the NPs, for example, description of the size, stability, charge and chemical composition. All active components, excipients and impurities have to be analyzed. Methods must be performed and validated according to good manufacturing procedure. Reproducibility of the production process must be documented and impurities and residual solvents must be below the levels detailed in guidelines or demonstrated to be nontoxic;

- General safety and toxicity studies: acute toxicity studies must be performed using at least three different doses in two species; most often rats and dogs are used. Repeat-dose toxicity studies with daily injections for 28 days are needed for an agent expected to be injected only once. The analyses include histological evaluations and analyses of a large number of
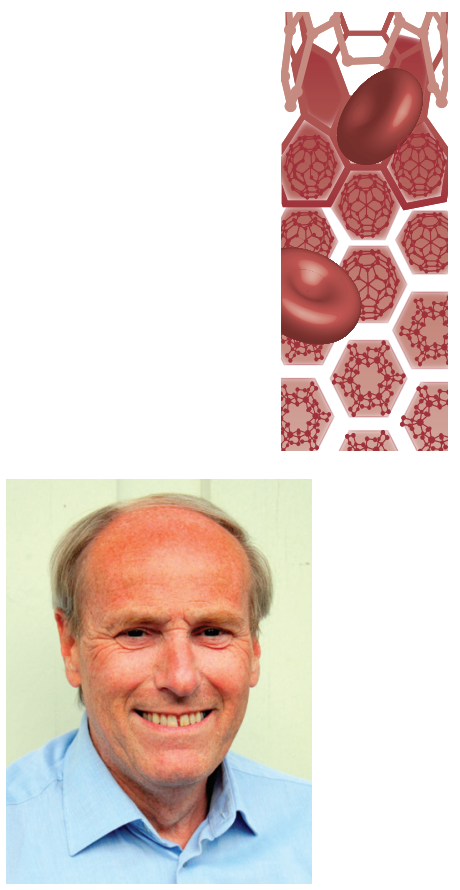

Tore Skotland

Author for correspondence:

Centre for Cancer Biomedicine, Faculty

Division Norwegian Radium Hospital,

University of Oslo, Oslo, Norway

and

Department of Biochemistry, Institute for Cancer Research, The Norwegian Radium Hospital, Oslo University Hospital,

Montebello, 0379 Oslo, Norway

Tel.: +4722781933

Fax: +4722781798

tore.skotland@rr-research.no

\section{Tore-Geir Iversen}

Author for correspondence: Centre for Cancer Biomedicine, Faculty Division Norwegian Radium Hospital, University of Oslo, Oslo, Norway and

Department of Biochemistry, Institute for Cancer Research, The Norwegian Radium Hospital, Oslo University Hospital, Montebello, 0379 Oslo, Norway

\section{Kirsten Sandvig}

Centre for Cancer Biomedicine, Faculty Division Norwegian Radium Hospital, University of Oslo, Oslo, Norway and

Department of Biochemistry, Institute for Cancer Research, The Norwegian Radium Hospital, Oslo University Hospital,

Montebello, 0379 Oslo, Norway and

Department of Biosciences, University of Oslo, 0316 Oslo, Norway

Future $\because$ Medicine part of 
parameters in blood and urine. Safety studies must be performed according to good laboratory practice (GLP);

- Specific toxicity studies: the specific toxicity studies needed depend upon the drug tested; include investigations of immunotoxicity, neurotoxicity, development and reproductive toxicity, genotoxicity and carcinogenicity;

- Administration, distribution, metabolism and excretion should be investigated for all constituents of the product. When such analyses are part of GLP safety studies the methods have to be performed and validated according to GLP. A key question is how accurate mass balance studies will be needed for a given NP-based product.

Currently, there is much focus on research with NPs that could be used for different imaging modalities or as theranostics - that is, products that can be used both for therapy and diagnosis [11]. Such NPs can certainly be of great use for preclinical studies, for example, to compare biodistribution and excretion of different constituents with the therapeutic efficacy of the product candidates. However, it is important to be aware of that intravenously injected products must prove beneficial for each specific clinical use in order to get market approval. The larger complexity of multifunctional NPs will make them more complicated and expensive to manufacture and analyze, and it is more difficult to obtain the necessary intellectual property owing to to the increased complexity of such products. There is no such product on the market today. Although discussions have been ongoing for several years, and guidelines exist, it is not clear, so far, how the authorities will deal with registration of the different types of new multifunctional NPs. Based upon statements from the regulatory authorities, one can expect that the procedures used for traditional drugs will be followed, meaning that each product will be handled as a separate issue. An important question is: how detailed the description will be needed for, for example, degradation and excretion of the constituents of such NPs?

The development of a traditional water-soluble lowmolecular weight contrast agent for medical imaging was estimated to cost close to US $\$ 100$ million 8 years ago, whereas the cost for development of therapeutics is considerably higher and varies from product to product [12]. The development cost of NP-based products would be expected to be higher than for traditional drugs since all substances in the product must be analyzed and more studies are needed to describe the biodistribution, degradation, excretion and safety of the injected particles.

\section{Risk/benefit evaluations}

The pharmaceutical industry performs a risk/benefit evaluation before starting development of new drugs. One can expect such evaluations to include at least the following issues:

- Market potential based on expected better therapeutic effect or less adverse reactions than products on the market or known to be under development;

- Expected cost for development up to marketing;

- Expected cost up to 'go' or 'no go' decision points for further development;

- Expected time for development of product until marketing;

- Risk of failure of product development due to technical issues;

- Risk of failure due to safety issues during preclinical evaluation;

- Risk of failure of product owing to efficacy or safety issues late in clinical studies - that is, after large investments;

- Risk of not satisfying formal regulatory demands, for example, lack of reproducibility of the manufactured product;

- Manufacturing costs versus market price;

- Risk of other companies coming to the market with an equal, cheaper or better product; thus not being able to reach the expected market share.

The cost versus benefit of adding targeting and imaging capabilities to NPs was recently discussed [13]. The high cost of such products and the fact that less adverse effects are accepted for an imaging agent than for a drug that can be life saving or life prolonging, makes it more attractive for pharmaceutical companies to go for development of NPs with a therapeutic potential rather than for imaging.

\section{Safety studies: importance of degradation \& excretion of NP constituents}

Very few safety studies have been performed long enough to address possible adverse effects due to degradation of the NPs. A recent detailed biodistribution and toxicology study with intravenous injection of cadmiun selenide/cadmium sulfide/zinc sulfide quantum dots into rhesus macaques (i.e., in a nonhuman primate model) is important for this discussion [14]. Surprisingly, these NPs did not give any toxicologi- 
cal effects in vivo although giving toxicological effects in vitro. Interestingly, the data reveal that the body is able to degrade such particles even if it has never been in contact with such substances [14]. However, the body was not able to excrete the degradation products, as $99 \%$ of the injected dose of cadmium was present 90 days after injection. Of all earlier studies with metal-based NPs, it is only iron oxide-based NPs that clearly have been shown to be degraded in the body, probably owing to the similarity with how the body degrades the iron oxide structures within the iron storage protein ferritin in lysosomes [7].

\section{"Several methods have been used to study biodistribution and degradation of NPs in animal studies. All methods have their advantages and disadvantages."}

We believe that the pharmaceutical industry will be sceptical to start development of new NP-based products made of nonendogenous potential toxic substances that accumulate in the body. This view is based upon the uncertainty regarding toxic effects and cost of development of such products compared with NPs that are degraded and excreted, but otherwise have a similar function. It is clear that safety studies with NPs that are slowly degraded and excreted, for example, such as the presently available quantum dots, will have to be performed for a very long time. Although there are no clear instructions or guidelines for how long-term studies need to be performed as part of the safety assessment, there is no doubt that with a product showing almost no excretion in a nonhuman primate 90 days after injection, preclinical safety studies must be performed for much longer than 90 days [10]. We believe it is important that researchers aiming at bringing new NP-based drugs into clinical use are aware of the much higher risk and cost associated with developing a NP-based product that is slowly degraded and excreted compared with NPs that are more rapidly excreted. The example from the MRI contrast agent field about the possible association between very small amounts of gadolinium $\left(\mathrm{Gd}^{3+}\right)$ retained in the body following injection of gadolinium-based contrast agents with the extremely serious disorder nephrogenic systemic fibrosis [15], is likely to make pharmaceutical companies even more cautious in investing into agents with a long retention time in the body.

There is a huge potential in developing safe NPbased products for medical use, for example, products that combine different drugs to combat the heterogeneous population of cancer cells within tumors. Products with a similar therapeutic effect, such as cancer drugs on the market, but with less adverse reactions would also be very useful. When developing NPbased drugs consisting of nonendogenous substances, it is important that the NPs are biodegradable and that their constituents are excreted as less than 50\% of the injected dose will be excreted as intact particles through the kidneys for NPs with a hydrodynamic diameter larger than 5-6 nm [7]. Furthermore, only a very minor fraction of the injected dose will home into the target. Studies with antibody-targeted radiotherapy show that much less than $1 \%$ of the injected dose homes to the patient's tumor [16], and passive targeting of NPs to tumors in animal models (due to the enhanced permeability and retention effect [17]) are normally $10-15 \%$ of the injected dose [6]. Keeping in mind that the tumor in such animal models may constitute approximately $5 \%$ of the body weight, it would be surprising if more than $5 \%$ of the injected dose will home into tumors in patients. Although the presence of metastases should be included into such calculations, it is likely that more than $95 \%$ of the injected dose of NPs with a hydrodynamic diameter larger than $10 \mathrm{~nm}$ will distribute to different parts of the body and be a potential source of toxicological effects $[6,7]$. Based on this discussion it is also clear that injection of NPs for image-guided surgery, followed by removal of the tumor will remove only a very small fraction of the injected particles [14].

Several methods have been used to study biodistribution and degradation of NPs in animal studies. All methods have their advantages and disadvantages. Using the fluorescent properties of NPs, for example, quantum dots, is a nice way to study the initial biodistribution. However, fluorescence cannot be used for quantitative studies of biodegradation, since the fluorescence of partly degraded particles is not known. Furthermore, when traditional fluorescent labeling is used, it is essential to know whether the label is still bound to the particles. Inductively coupled plasmamass spectrometry is a very sensitive technique for quantification of metals in biological samples; however, it does not tell if the metal is present in particles or as released metal ions. Radioactive labeling may be used both for imaging and for quantitative analyses of biological samples. However, care should be taken to ensure that the radioactivity measured gives the true picture of the metabolism and excretion of particles - that is, not using isotopes that just dissociate from the particles or the chelates they were bound to or that the isotope-chelate complex is split from the NP and thus do not represent the localization of the particles. The best approach will be to combine some of these methods, and the method of choice will depend upon the particles studied. 
The way forward: new studies \& improved quality

In order to trust the outcome of biological studies, one needs to improve the characterization of NPs used [18]; it is necessary to include many methods in order to perform the analyses and satisfy the criteria listed in the bullet points in the 'Regulatory requirements \& costs for bringing new drugs to the market' section above [9,19]. In addition, the quality of in vitro cell studies has to be improved in order to trust the conclusions drawn [20]. Surprisingly, many studies have been performed in the absence of serum or plasma proteins in the medium, although the presence of such proteins obviously is important in order to mimic in vivo conditions. In many studies, it is concluded that the NPs do not have any toxic effects after cellular uptake, whereas the data presented do not actually discriminate between particles just adsorbed to the cells and taken up into the cells. We have discussed these issues in a review article, where we also present a tool box to help researchers improve the quality of studies on uptake and intracellular transport of NPs [20].

Protein binding to NPs has been shown to be important for in vitro cellular uptake of NPs [21,22]. Further studies are needed to understand the importance of the protein 'corona' for biodistribution and safety of NPs following intravenous injection. Modification of NPs with polyethylenglycol has been shown to change the protein binding to the particles, increase the circulation time and change the biodistribution of the NPs [23], but more investigations are needed to learn which effects this will have on the long-term toxicology [7]. Increased knowledge about how safety of NPs is linked to materialintrinsic properties is essential for further development of nanomedicine $[24,25]$. Often researchers claim that the NPs studied are biocompatible or biodegradable without providing convincing scientific documentation for their statements [6].

The discussion above illustrates that the nanomedicine community has to overcome many challenges

\section{References}

1 Petros RA, DeSimone JM. Strategies in the design of nanoparticles for therapeutic applications. Nat. Rev. Drug Discov. 9, 615-627 (2010).

2 Parveen S, Misra R, Sahoo SK. Nanoparticles: a boon to drug delivery, therapeutics, diagnostics and imaging. Nanomedicine 8, 147-166 (2012).

3 Nichols JW, Bae YH. Odyssey of a cancer nanoparticle: from injection site to site of action. Nano Today 7, 606-618 (2012).

4 Gozzard D. When is high-dose intravenous iron repletion needed? Assessing new treatment options. Drug Des. Devel. Ther. 5, 51-60 (2011). over the next few years. It is important to improve the quality of research both for the sake of reputation of the nanomedicine field and to bring the field forward in an optimal way [18]. The solution to overcome these problems seems obvious to us: it is necessary to establish more crossfunctional collaboration between experts in the different relevant scientific areas, such as synthesis and manufacturing of NPs; characterization of NPs in the absence and presence of proteins; in vitro studies of cellular uptake, intracellular transport, biodegradation and cellular toxicity; in vivo preclinical studies using relevant animal models to study efficacy and biodistribution; bioanalysis to quantify NP-containing substances and metabolites in biological tissues, urine and feces; imaging modalities, such as fluorescence imaging, MRI and nuclear imaging techniques, such as PET/singlephoton emission computerized tomography; safety studies, including, immunological investigations and other specific safety studies as detailed above; clinical studies involving experts knowing the clinical need for new products. Hopefully, during the next few years, we will see much more of such interdisciplinary collaborations, which can improve quality of the studies, provide us with new methods/protocols and conclusions that can be trusted and used to build a solid fundament for future development of new NP-based drugs.

\section{Financial \& competing interests disclosure}

This work was supported by the Research Council of Norway through the NANO2021 project (project number 228200) and through the Centre of Excellence funding scheme (project number 179571), the South-Eastern Norway Regional Health Authority and the Norwegian Cancer Society. The authors have no other relevant affiliations or financial involvement with any organization or entity with a financial interest in or financial conflict with the subject matter or materials discussed in the manuscript apart from those disclosed.

No writing assistance was utilized in the production of this manuscript.
5 Corot C, Robert P, Idee JM, Port M. Recent advances in iron oxide nanocrystal technology for medical imaging. $A d v$. Drug Deliv. Rev. 58, 1471-1504 (2006).

6 Duncan R, Gaspar R. Nanomedicine(s) under the Microscope. Mol. Pharm. 8, 2101-2141 (2011).

7 Skotland T, Iversen TG, Sandvig K. New metal-based nanoparticles for intravenous use: requirements for clinical success with focus on medical imaging. Nanomedicine 6, 730-737 (2010).

8 Chauhan VP, Jain RK. Strategies for advancing cancer nanomedicine. Nat. Mater. 12, 958-962 (2013).

9 Li C. A targeted approach to cancer imaging and therapy. Nat. Mater. 13, 110-115 (2014). 
ance for Industry. Developing medical imaging drug and biological products: part 1: conducting safety assessments.

www.fda.gov/downloads/Drugs/

GuidanceComplianceRegulatoryInformation/Guidance/ ucm078930.pdf

11 Lee DE, Koo H, Sun IC, Ryu JH, Kim K, Kwon IC. Multifunctional nanoparticles for multimodal imaging and theragnosis. Chem. Soc. Rev. 41, 2656-2672 (2012).

12 Nunn AD. The cost of developing imaging agents for routine clinical use. Invest. Radiol. 41, 206-212 (2006).

13 Cheng Z, Al ZA, Hui JZ, Muzykantov VR, Tsourkas A. Multifunctional nanoparticles: cost versus benefit of adding targeting and imaging capabilities. Science 338, 903-910 (2012).

14 Ye L, Yong KT, Liu L et al. A pilot study in non-human primates shows no adverse response to intravenous injection of quantum dots. Nat. Nanotechnol. 7, 453-458 (2012).

15 Rofsky NM, Sherry AD, Lenkinski RE. Nephrogenic systemic fibrosis: a chemical perspective. Radiology 247, 608-612 (2008).

16 Goldenberg DM. Advancing role of radiolabeled antibodies in the therapy of cancer. Cancer Immunol. Immunother. 52, 281-296 (2003).

17 Fang J, Nakamura H, Maeda $\mathrm{H}$. The EPR effect: Unique features of tumor blood vessels for drug delivery, factors involved, and limitations and augmentation of the effect. Adv. Drug Deliv. Rev. 63, 136-151 (2011).
18 Editorial: Join the dialogue. Nat. Nanotechnol. 7, 545-545 (2012).

19 Crist RM, Grossman JH, Patri AK et al. Common pitfalls in nanotechnology: lessons learned from NCI's nanotechnology characterization laboratory. Integr. Biol. (Camb.) 5, 66-73 (2013).

20 Iversen TG, Skotland T, Sandvig K. Endocytosis and intracellular transport of nanoparticles: present knowledge and need for future studies. Nano Today 6, 176-185 (2011).

21 Monopoli MP, Aberg C, Salvati A, Dawson KA. Biomolecular coronas provide the biological identity of nanosized materials. Nat. Nanotechnol. 7, 779-786 (2012).

22 Tenzer S, Docter D, Kuharev J et al. Rapid formation of plasma protein corona critically affects nanoparticle pathophysiology. Nat. Nanotechnol. 8, 772-781 (2013).

23 Schipper ML, Iyer G, Koh AL et al. Particle size, surface coating, and PEGylation influence the biodistribution of quantum dots in living mice. Small 5, 126-134 (2009).

24 Fadeel B, Feliu N, Vogt C, Abdelmonem AM, Parak WJ. Bridge over troubled waters: understanding the synthetic and biological identities of engineered nanomaterials. Wiley Interdiscip. Rev. Nanomed. Nanobiotechnol. 5, 111-129 (2013).

25 Cho EJ, Holback H, Liu KC, Abouelmagd SA, Park J, Yeo Y. Nanoparticle characterization: state of the art, challenges, and emerging technologies. Mol. Pharm. 10, 2093-2110 (2013). 